\title{
molecules
}

ISSN 1420-3049

http://www.mdpi.org

\section{Functionalization of 6-Nitrobenzo[1,3]dioxole with Carbonyl Compounds via TDAE Methodology}

\section{Ouassila Amiri-Attou, Thierry Terme and Patrice Vanelle*}

Laboratoire de Chimie Organique Pharmaceutique, CNRS-UMR 6517, Faculté de Pharmacie, 27 Bd Jean Moulin, 13385 Marseille Cx 5, France

* Author to whom correspondence should be addressed.

E-mail: patrice.vanelle@pharmacie.univ-mrs.fr

Received: 31 January 2005 / Accepted: 5 February 2005 / Published: 13 May 2005

\begin{abstract}
We report herein the synthesis of substituted 2-(6-nitrobenzo[1,3]dioxol-5-yl)-1aryl ethanols and 2-(6-nitrobenzo[1,3]dioxol-5-yl)-propionic acid ethyl esters from the reaction of 5-chloromethyl-6-nitrobenzo[1,3]dioxole with various aromatic carbonyl and $\alpha$ carbonyl ester derivatives using the tetrakis(dimethylamino)ethylene (TDAE) methodology.
\end{abstract}

Keywords: TDAE, Nitrobenzo[1,3]dioxole, Arylethanols, Carbonyl derivatives.

\section{Introduction}

Many therapeutically important arylethanolamines [1] (isoprenaline, salbutamol, etc.) have a wide range of activities, including $\alpha, \beta$-adrenoreceptor and monoamine reuptake binding affinities. Regulation of these sites has been sought in the treatment of high blood pressure, asthma, diabetes, anxiety, depression, etc. Consequently, in light of these therapeutically important activities, this class of compounds has attracted the attention of organic chemists.

\section{Scheme 1.}<smiles>CN(C)CC(O)c1ccc(O)c(O)c1</smiles>

Isoprenaline

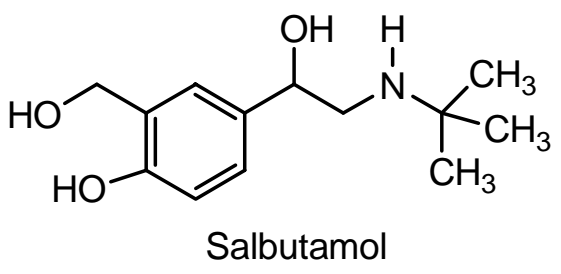

Salbutamol 
Tetrakis(dimethylamino)ethylene (TDAE) is a reducing agent which reacts with halogenated derivatives to generate an anion under mild conditions via a single electron transfer (SET) [2-4]. We have recently shown that TDAE could generate a nitrobenzyl carbanion from $o$ - or $p$-nitrobenzyl chloride which is able to react with various electrophiles such as aromatic aldehydes [5] or pyruvate derivatives [6]. In continuation of our program directed toward the study of single electron transfer reactions of bioreductive alkylating agents and the preparation of new potentially bioactive compounds [7-9], we report herein the synthesis of substituted 2-(6-nitrobenzo[1,3]dioxol-5-yl)-1-arylethanols and 2-(6-nitrobenzo[1,3]dioxol-5-yl)-propionic acid ethyl esters from the reactions of 5-chloromethyl-6nitrobenzo[1,3]dioxole with various aromatic carbonyl and $\alpha$-carbonyl ester derivatives using the TDAE methodology. This reactivity was the first step of the synthesis of a new class of arylethanolamine analogs (deprotection of dioxole and reduction of nitro group).

\section{Results and Discussion}

(6-Nitrobenzo[1,3]dioxol-5-yl)methanol (1) treated with thionyl chloride in dichloromethane gives 5-chloromethyl-6-nitrobenzo[1,3]dioxole (2) in 95\% yield [10] (Scheme 2).

\section{Scheme 2.}

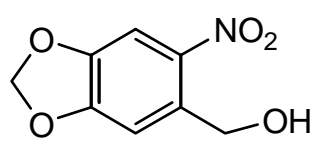

1

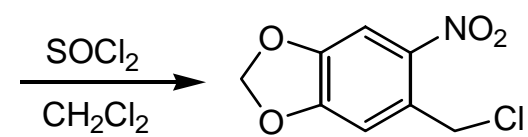

$295 \%$

This chloro derivative $\mathbf{2}$ is a good candidate for generating a 6-nitrobenzo[1,3]dioxole anion in an easy, original and mild method using TDAE. Thus, derivative 2 reacts with aromatic aldehydes 3a-g in the presence of TDAE to form substituted 2-(6-nitrobenzo[1,3]dioxol-5-yl)-1-arylethanols $\mathbf{4 a - g}$ in moderate to good yields, as shown in Scheme 3 and Table 1. As shown in the p-nitrobenzyl series [7], aldehydes with electron-withdrawing groups such as nitro (68 and 56\%) or trifluoromethyl (56\%) are more reactive than aldehydes substituted with an electron-donor group such as methyl (for example, 3d, 23\%). Under the same experimental conditions, we have studied the reaction of derivative 2 with 4-nitroacetophenone (3h) in presence of TDAE. After $2 \mathrm{~h}$ at room temperature, TLC analysis (dichloromethane as eluent) clearly showed that $\mathbf{2}$ was not totally consumed and the starting material was only totally consumed after $24 \mathrm{~h}$ of reaction at room temperature. This fact was in accord with Médebielle's results [3] and may be explained by the difference of reactivity between aldehydes and ketones. Moreover, after the reaction with aromatic carbonyl derivatives, we have investigated the reaction of the 6 -nitrobenzo[1,3]dioxole anion with $\alpha$-keto-ester derivatives as methyl pyruvate (3i), ethyl glyoxylate (3j), diethyl ketomalonate (3k) and methyl isatin (3l). The reaction of $\mathbf{2}$ in presence of TDAE with these electrophiles furnished the corresponding $\alpha$-hydroxy-ester $\mathbf{4} \mathbf{i}-\mathbf{k}$ or $\alpha$-hydroxy-lactam $4 \mathbf{l}$ derivatives in moderate to good yields (43-84\%) as shown in Table 1.

Scheme 3.

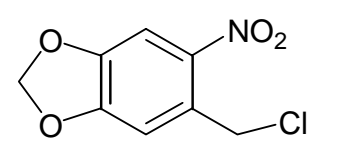

2

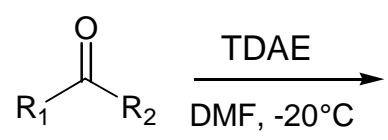

3a-I

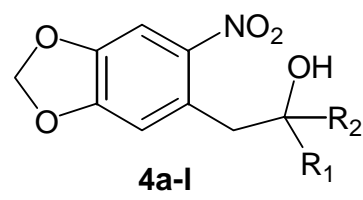

4a-I 
Table 1. Reaction of chloride 2 and carbonyl compounds using TDAE ${ }^{\mathrm{a}}$.

\begin{tabular}{|c|c|c|c|c|c|}
\hline Entry & $\begin{array}{l}\text { Carbonyl } \\
\text { compound }\end{array}$ & Product & $\begin{array}{l}\text { Product } \\
\text { number }\end{array}$ & $\begin{array}{l}\text { Reaction time } \\
\text { (h) }\end{array}$ & $\begin{array}{l}\text { Yield } \\
(\%)^{b}\end{array}$ \\
\hline $\mathbf{a}$ & & & $4 a$ & 2 & 68 \\
\hline b & & & $4 b$ & 2 & 28 \\
\hline c & & & $4 c$ & 2 & 40 \\
\hline d & & & 4d & 2 & 23 \\
\hline e & & & $4 e$ & 2 & 56 \\
\hline $\mathbf{f}$ & & & $4 f$ & 2 & 56 \\
\hline g & & & $4 g$ & 2 & 34 \\
\hline $\mathbf{h}$ & & & $4 h$ & 24 & 40 \\
\hline $\mathbf{i}$ & & & $4 i$ & 2 & 75 \\
\hline $\mathbf{j}$ & & & $4 j$ & 2 & 84 \\
\hline $\mathbf{k}$ & & & $4 k$ & 2 & 63 \\
\hline
\end{tabular}


Table 1. Cont.

\begin{tabular}{|c|c|c|c|c|c|}
\hline Entry & $\begin{array}{l}\text { Carbonyl } \\
\text { compound }\end{array}$ & Product & $\begin{array}{l}\text { Product } \\
\text { number }\end{array}$ & $\begin{array}{l}\text { Reaction time } \\
\text { (h) }\end{array}$ & $\begin{array}{l}\text { Yield } \\
(\%)^{b}\end{array}$ \\
\hline I & & & 41 & 2 & 43 \\
\hline
\end{tabular}

${ }^{\text {a }}$ All the reactions are performed using 3 equivalents of carbonyl compounds 3a-l, 1 equivalent of chloride 2 and 1 equivalent of TDAE in anhydrous DMF stirred at $-20^{\circ} \mathrm{C}$ for $1 \mathrm{~h}$ and then warmed up to room temperature. ${ }^{\mathrm{b}} \%$ Yield relative to chloride 2.

The mechanism of these reactions appears to proceed via an initially formed charge-transfer complex between chloride 2 and TDAE, followed by stepwise single-electron transfers of two electrons from TDAE to chloride $\mathbf{2}$ to form $\mathrm{TDAE}^{2+}$ and the anion of $\mathbf{2}$ which reacts by nucleophilic addition on carbonyl compounds 3a-l.

\section{Conclusions}

We have demonstrated in this work that 5-chloromethyl-6-nitrobenzo[1,3]dioxole (2) and aromatic carbonyl or pyruvate derivatives react in the presence of TDAE to afford diaryl alcohols, $\alpha$-hydroxy esters and $\alpha$-hydroxy lactam derivatives in moderate to good yields. This method, using TDAE, is an easy, original and mild method to prepare functionalized $o$-nitrobenzyl anions in situ. This approach was found to be milder compared with the classical Reformatsky reaction, where activated zinc with added catalyst is often needed. The preparation of a new class of arylethanolamine analogs (after deprotection of dioxole ring and reduction of nitro group) and the pharmacological evaluation of all intermediates are under active investigation.

\section{Experimental}

\section{General}

Melting points were determined on a Büchi B-540 apparatus and are uncorrected. Elemental analyses were performed by the Centre de Microanalyses of the University of Aix-Marseille 3. Both ${ }^{1} \mathrm{H}$ - and ${ }^{13} \mathrm{C}$-NMR spectra were determined on a Bruker ARX 200 spectrometer. The ${ }^{1} \mathrm{H}$ - chemical shifts were reported as parts per million downfield from tetramethylsilane $\left(\mathrm{Me}_{4} \mathrm{Si}\right)$, and the ${ }^{13} \mathrm{C}$ chemical shifts were referenced to the solvent peak of $\mathrm{CDCl}_{3}(76.9 \mathrm{ppm})$. Silica gel 60 (Merck, 230400 mesh) was used for column chromatography. Thin-layer chromatography was performed with Merck 60F-254 (0.25 mm layer thickness) silica gel plates.

General procedure for the reaction of chloride $\mathbf{2}$ and aromatic carbonyl derivatives 3a-h, $\alpha$-carbonyl esters 3i-j, ketomalonate $\mathbf{3 k}$ and keto-lactam $\mathbf{3 l}$ using TDAE

A solution of $2(0.30 \mathrm{~g}, 1.36 \mathrm{mmol})$ in anhydrous DMF $(7 \mathrm{~mL})$ and the corresponding carbonyl derivative 3a-l (9 mmol, 3 equiv.) were placed under nitrogen at $-20^{\circ} \mathrm{C}$ in a two-necked flask equipped with a silica-gel drying tube and a nitrogen inlet. The solution was stirred and kept at this temperature 
for $30 \mathrm{~min}$ and then the TDAE $(0.27 \mathrm{~g}, 1.36 \mathrm{mmol})$ was added dropwise via a syringe. A red color immediately developed with the formation of a white fine precipitate. The solution was vigorously stirred at $-20^{\circ} \mathrm{C}$ for $1 \mathrm{~h}$ and then warmed up to room temperature for $2 \mathrm{~h}$ ( $24 \mathrm{~h}$ for reaction with p-nitroacetophenone 3h). After this time TLC analysis $\left(\mathrm{CH}_{2} \mathrm{Cl}_{2}\right.$ as eluent) clearly showed that 2 was totally consumed. The orange-red turbid solution was filtered (to remove the octamethyloxamidinium dichloride) and hydrolysed with $\mathrm{H}_{2} \mathrm{O}(80 \mathrm{~mL})$. The aqueous solution was extracted with toluene $(3 \mathrm{x}$ $40 \mathrm{~mL}$ ), the combined organic layers washed with $\mathrm{H}_{2} \mathrm{O}(3 \times 40 \mathrm{~mL})$ and dried over $\mathrm{MgSO}_{4}$. Evaporation of the solvent left an orange viscous liquid as crude product. Purification by silica gel chromatography $\left(\mathrm{CH}_{2} \mathrm{Cl}_{2}\right)$ gave the corresponding arylethanol, $\alpha$-hydroxy ester or $\alpha$-hydroxy lactam derivatives.

2-(6-Nitrobenzo[1,3] dioxol-5-yl)-1-(4-nitrophenyl)ethanol (4a): Orange solid; mp $162{ }^{\circ} \mathrm{C}$ (ethyl alcohol); ${ }^{1} \mathrm{H}-\mathrm{NMR}\left(\mathrm{CDCl}_{3}\right) \delta: 2.36$ (bs, $\left.1 \mathrm{H}, \mathrm{OH}\right), 3.01$ (dd, $J_{\mathrm{AB}}=13.6 \mathrm{~Hz}$ and $J=8.9 \mathrm{~Hz}, 1 \mathrm{H}, \mathrm{CH}_{2}$ ), $3.43\left(\mathrm{dd}, J_{\mathrm{AB}}=13.6 \mathrm{~Hz}\right.$ and $J=3.5 \mathrm{~Hz}, 1 \mathrm{H}, \mathrm{CH}_{2}$ ), 5.18 (dd, $J=8.9 \mathrm{~Hz}$ and $J=3.5 \mathrm{~Hz}, 1 \mathrm{H}, \mathrm{CH}$ ), 6.12 (s, $\left.2 \mathrm{H}, \mathrm{CH}_{2}\right), 6.73\left(\mathrm{~s}, 1 \mathrm{H}, \mathrm{H}_{\mathrm{Ar}}\right), 7.56\left(\mathrm{~s}, 1 \mathrm{H}, \mathrm{H}_{\mathrm{Ar}}\right), 7.63\left(\mathrm{~d}, J=8.6 \mathrm{~Hz}, 2 \mathrm{H}, 2 \mathrm{xH}_{\mathrm{Ar}}\right), 8.23(\mathrm{~d}, J=8.6 \mathrm{~Hz}$, 2H, $\left.2 \mathrm{xH}_{\mathrm{Ar}}\right) ;{ }^{13} \mathrm{C}-\mathrm{NMR}\left(\mathrm{CDCl}_{3}\right) \delta$ : $43.8\left(\mathrm{CH}_{2}\right), 73.4(\mathrm{CH}), 103.0\left(\mathrm{CH}_{2}\right), 105.9(\mathrm{CH}), 112.1(\mathrm{CH}), 123.8$ (2xCH), 126.5 (2xCH), 129.9 (C), 147.2 (C), 147.5 (C), 151.0 (C), 151.7 (C), 158.3 (C); Anal. Calcd for $\mathrm{C}_{15} \mathrm{H}_{12} \mathrm{~N}_{2} \mathrm{O}_{7}$ : C, 54.22; H, 3.64; N, 8.43. Found: C, 54.17; H, 3.50; N, 8.40.

1-(4-Chlorophenyl)-2-(6-nitrobenzo[1,3]dioxol-5-yl)ethanol (4b): Orange solid; mp $109{ }^{\circ} \mathrm{C}$ (ethyl alcohol); ${ }^{1} \mathrm{H}-\mathrm{NMR}\left(\mathrm{CDCl}_{3}\right) \delta: 1.67$ (bs, $\left.1 \mathrm{H}, \mathrm{OH}\right), 3.05$ (dd, $J_{\mathrm{AB}}=13.6 \mathrm{~Hz}$ and $J=8.9 \mathrm{~Hz}, 1 \mathrm{H}, \mathrm{CH}_{2}$ ), $3.35\left(\mathrm{dd}, J_{\mathrm{AB}}=13.6 \mathrm{~Hz}\right.$ and $\left.J=3.7 \mathrm{~Hz}, 1 \mathrm{H}, \mathrm{CH}_{2}\right), 5.13(\mathrm{dd}, J=8.9 \mathrm{~Hz}$ and $J=3.7 \mathrm{~Hz}, 1 \mathrm{H}, \mathrm{CH}), 6.10$ (s, $\left.2 \mathrm{H}, \mathrm{CH}_{2}\right), 6.72\left(\mathrm{~s}, 1 \mathrm{H}, \mathrm{H}_{\mathrm{Ar}}\right), 7.35\left(\mathrm{~m}, 4 \mathrm{H}, 4 \mathrm{xH}_{\mathrm{Ar}}\right), 7.53\left(\mathrm{~s}, 1 \mathrm{H}, \mathrm{H}_{\mathrm{Ar}}\right) ;{ }^{13} \mathrm{C}-\mathrm{NMR}\left(\mathrm{CDCl}_{3}\right) \delta: 43.7$ $\left(\mathrm{CH}_{2}\right), 73.6(\mathrm{CH}), 102.9\left(\mathrm{CH}_{2}\right), 105.8(\mathrm{CH}), 112.1(\mathrm{CH}), 127.0(2 \mathrm{xCH}), 128.7(2 \times \mathrm{CH}), 130.4(\mathrm{C})$, 133.5 (C), 142.4 (C), 143.3 (C), 147.0 (C), 151.5 (C); Anal. Calcd for $\mathrm{C}_{15} \mathrm{H}_{12} \mathrm{ClNO}_{5}$ : C, 56.00; H, 3.76; N, 4.35. Found: C, 56.29; H, 3.77; N, 4.43.

4-[1-Hydroxy-2-(6-nitrobenzo[1,3]dioxol-5-yl)-ethyl]benzonitrile (4c): Yellow solid; mp $166{ }^{\circ} \mathrm{C}$ (dichloromethane); ${ }^{1} \mathrm{H}-\mathrm{NMR}\left(\mathrm{CDCl}_{3}\right) \delta: 2.32$ (bs, $\left.1 \mathrm{H}, \mathrm{OH}\right), 2.99$ (dd, $J_{\mathrm{AB}}=13.5 \mathrm{~Hz}$ and $J=9.0 \mathrm{~Hz}$, $1 \mathrm{H}, \mathrm{CH}_{2}$ ), 3.40 (dd, $J_{\mathrm{AB}}=13.5 \mathrm{~Hz}$ and $J=3.5 \mathrm{~Hz}, 1 \mathrm{H}, \mathrm{CH}_{2}$ ), 5.12 (dd, $J=9.0 \mathrm{~Hz}$ and $J=3.5 \mathrm{~Hz}, 1 \mathrm{H}$, $\mathrm{CH}$ ), 6.12 (s, 2H, $\mathrm{CH}_{2}$ ), $6.72\left(\mathrm{~s}, 1 \mathrm{H}, \mathrm{H}_{\mathrm{Ar}}\right), 7.55$ (s, $\left.1 \mathrm{H}, \mathrm{H}_{\mathrm{Ar}}\right), 7.57$ (d, $J=8.4 \mathrm{~Hz}, 2 \mathrm{H}, 2 \mathrm{xH}_{\mathrm{Ar}}$ ), 7.67 (d, $J$ $\left.=8.4 \mathrm{~Hz}, 2 \mathrm{H}, 2 \mathrm{xH}_{\mathrm{Ar}}\right) ;{ }^{13} \mathrm{C}-\mathrm{NMR}\left(\mathrm{CDCl}_{3}\right) \delta: 43.7\left(\mathrm{CH}_{2}\right), 73.5(\mathrm{CH}), 103.0\left(\mathrm{CH}_{2}\right), 105.9(\mathrm{CH}), 111.5$ (C), $112.1(\mathrm{CH}), 118.7$ (C), 126.3 (2xCH), 130.0 (C), 132.4 (2xCH), 143.3 (C), 147.2 (C), $149.1(\mathrm{C})$, 151.7 (C); Anal. Calcd for $\mathrm{C}_{16} \mathrm{H}_{12} \mathrm{~N}_{2} \mathrm{O}_{5}$ : C, 61.54; H, 3.87; N, 8.97. Found: C, 61.63; H, 3.83; N, 8.67.

2-(6-Nitrobenzo[1,3]dioxol-5-yl)-1-p-tolylethanol (4d): Brown solid; mp $126{ }^{\circ} \mathrm{C}$ (diethyl ether); ${ }^{1} \mathrm{H}$ NMR $\left(\mathrm{CDCl}_{3}\right) \delta: 1.89$ (bs, $\left.1 \mathrm{H}, \mathrm{OH}\right), 2.36$ (s, 3H, $\mathrm{CH}_{3}$ ), 3.10 (dd, $J_{\mathrm{AB}}=13.5 \mathrm{~Hz}$ and $J=9.0 \mathrm{~Hz}, 1 \mathrm{H}$, $\mathrm{CH}_{2}$ ), 3.35 (dd, $J_{\mathrm{AB}}=13.5 \mathrm{~Hz}$ and $J=3.7 \mathrm{~Hz}, 1 \mathrm{H}, \mathrm{CH}_{2}$ ), 4.98 (dd, $J=9.0 \mathrm{~Hz}$ and $J=3.7 \mathrm{~Hz}, 1 \mathrm{H}, \mathrm{CH}$ ), 6.09 (s, $2 \mathrm{H}, \mathrm{CH}_{2}$ ), 6.75 (s, $1 \mathrm{H}, \mathrm{H}_{\mathrm{Ar}}$ ), 7.18 (d, $J=8.0 \mathrm{~Hz}, 2 \mathrm{H}, 2 \mathrm{xH}_{\mathrm{Ar}}$ ), 7.31 (d, $J=8.0 \mathrm{~Hz}, 2 \mathrm{H}, 2 \mathrm{xH}_{\mathrm{Ar}}$ ), $7.51\left(\mathrm{~s}, 1 \mathrm{H}, \mathrm{H}_{\mathrm{Ar}}\right) ;{ }^{13} \mathrm{C}-\mathrm{NMR}\left(\mathrm{CDCl}_{3}\right) \delta$ : $21.1\left(\mathrm{CH}_{3}\right), 43.5\left(\mathrm{CH}_{2}\right), 74.2(\mathrm{CH}), 102.8\left(\mathrm{CH}_{2}\right), 105.7(\mathrm{CH})$, 112.1 (CH), 125.5 (2xCH), 129.3 (2xCH), 130.9 (C), 137.5 (C), 140.9 (C), 143.4 (C), 146.8 (C), 151.4 (C); Anal. Calcd for $\mathrm{C}_{16} \mathrm{H}_{15} \mathrm{NO}_{5}$ : C, 63.78; H, 5.02; N, 4.65. Found: C, 63.77; H, 5.05; N, 4.58. 
2-(6-Nitrobenzo[1,3] dioxol-5-yl)-1-(2-nitrophenyl)ethanol (4e): Orange solid; mp $135{ }^{\circ} \mathrm{C}$ (ethyl alcohol); ${ }^{1} \mathrm{H}-\mathrm{NMR}\left(\mathrm{CDCl}_{3}\right)$ \&: 3.22 (bs, $\left.1 \mathrm{H}, \mathrm{OH}\right), 3.30$ (dd, $J_{\mathrm{AB}}=13.9 \mathrm{~Hz}$ and $J=3.5 \mathrm{~Hz}, 1 \mathrm{H}, \mathrm{CH}_{2}$ ), $3.48\left(\mathrm{dd}, J_{\mathrm{AB}}=13.9 \mathrm{~Hz}\right.$ and $J=9.1 \mathrm{~Hz}, 1 \mathrm{H}, \mathrm{CH}_{2}$ ), 5.51 (dd, $J=9.1 \mathrm{~Hz}$ and $\left.J=3.5 \mathrm{~Hz}, 1 \mathrm{H}, \mathrm{CH}\right), 6.11$ (s, 2H, $\mathrm{CH}_{2}$ ), 6.95 (s, $\left.1 \mathrm{H}, \mathrm{H}_{\mathrm{Ar}}\right), 7.43$ (s, $\left.1 \mathrm{H}, \mathrm{H}_{\mathrm{Ar}}\right), 7.48$ (m, 1H, $\mathrm{H}_{\mathrm{Ar}}$ ), 7.69 (m, 1H, $\mathrm{H}_{\mathrm{Ar}}$ ), 7.92 (m, 2H, 2xH $\left.\mathrm{Ar}_{\mathrm{Ar}}\right) ;{ }^{13} \mathrm{C}-\mathrm{NMR}\left(\mathrm{CDCl}_{3}\right)$ \&: $40.7\left(\mathrm{CH}_{2}\right), 70.9(\mathrm{CH}), 102.9\left(\mathrm{CH}_{2}\right), 105.5(\mathrm{CH}), 111.1(\mathrm{CH}), 124.6$ (CH), $128.4(\mathrm{CH}), 128.5(\mathrm{CH}), 129.7(\mathrm{C}), 133.8(\mathrm{CH}), 139.4(\mathrm{C}), 147.0(\mathrm{C}), 147.6(\mathrm{C}), 151.8(\mathrm{C})$, 158.6 (C); Anal. Calcd for $\mathrm{C}_{15} \mathrm{H}_{12} \mathrm{~N}_{2} \mathrm{O}_{7}$ : C, 54.22; H, 3.64; N, 8.43. Found: C, 54.35; H, 3.69; N, 8.15.

2-(6-Nitrobenzo[1,3]dioxol-5-yl)-1-(2-trifluoromethylphenyl)ethanol (4f): Brown solid; mp $102{ }^{\circ} \mathrm{C}$ (ethyl alcohol); ${ }^{1} \mathrm{H}-\mathrm{NMR}\left(\mathrm{CDCl}_{3}\right) \delta: 2.62$ (bs, $\left.1 \mathrm{H}, \mathrm{OH}\right), 3.09$ (dd, $J_{\mathrm{AB}}=14.0 \mathrm{~Hz}$ and $J=3.9 \mathrm{~Hz}, 1 \mathrm{H}$, $\mathrm{CH}_{2}$ ), 3.48 (dd, $J_{\mathrm{AB}}=14.0 \mathrm{~Hz}$ and $J=9.3 \mathrm{~Hz}, 1 \mathrm{H}, \mathrm{CH}_{2}$ ), 5.34 (dd, $J=9.3 \mathrm{~Hz}$ and $J=3.9 \mathrm{~Hz}, 1 \mathrm{H}, \mathrm{CH}$ ), $6.08\left(\mathrm{~s}, 2 \mathrm{H}, \mathrm{CH}_{2}\right), 6.73\left(\mathrm{~s}, 1 \mathrm{H}, \mathrm{H}_{\mathrm{Ar}}\right), 7.40\left(\mathrm{~m}, 2 \mathrm{H}, 2 \mathrm{xH}_{\mathrm{Ar}}\right), 7.60\left(\mathrm{~m}, 2 \mathrm{H}, 2 \mathrm{xH}_{\mathrm{Ar}}\right), 7.80\left(\mathrm{~m}, 1 \mathrm{H}, \mathrm{H}_{\mathrm{Ar}}\right) ;{ }^{13} \mathrm{C}-$ NMR $\left(\mathrm{CDCl}_{3}\right) \delta$ : $41.6\left(\mathrm{CH}_{2}\right), 70.1(\mathrm{CH}), 102.8\left(\mathrm{CH}_{2}\right), 105.5(\mathrm{CH}), 111.0(\mathrm{CH}), 125.4(\mathrm{CH}), 126.6(\mathrm{C})$, 127.0 (C), 127.8 (2xCH), 129.5 (C), 132.4 (CH), 142.7 (C), 144.3 (C), 146.8 (C), 151.5 (C); Anal. Calcd for $\mathrm{C}_{16} \mathrm{H}_{12} \mathrm{~F}_{3} \mathrm{NO}_{5}$ : C, 54.09; H, 3.40; N, 3.94. Found: C, 53.82; H, 3.21; N, 3.92.

1,2-Bis-(6-nitrobenzo[1,3]dioxol-5-yl)ethanol (4g): Yellow solid; mp $230{ }^{\circ} \mathrm{C}$ (chloroform); ${ }^{1} \mathrm{H}-\mathrm{NMR}$ $\left(\mathrm{DMSO}_{-} \mathrm{d}_{6}\right) \delta: 3.07$ (dd, $J_{\mathrm{AB}}=13.6 \mathrm{~Hz}$ and $J=4.4 \mathrm{~Hz}, 1 \mathrm{H}, \mathrm{CH}_{2}$ ), 3.35 (dd, $J_{\mathrm{AB}}=13.6 \mathrm{~Hz}$ and $J=8.2$ $\mathrm{Hz}, 1 \mathrm{H}, \mathrm{CH}_{2}$ ), 5.27 (m, 1H, CH), 5.72 (bs, 1H, OH), 6.19 (s, 2H, $\left.\mathrm{CH}_{2}\right), 6.22\left(\mathrm{~s}, 2 \mathrm{H}, \mathrm{CH}_{2}\right), 6.90$ (s, 1H, $\mathrm{H}_{\mathrm{Ar}}$ ), 7.15 (s, 1H, $\left.\mathrm{H}_{\mathrm{Ar}}\right), 7.51\left(\mathrm{~s}, 1 \mathrm{H}, \mathrm{H}_{\mathrm{Ar}}\right), 7.52\left(\mathrm{~s}, 1 \mathrm{H}, \mathrm{H}_{\mathrm{Ar}}\right) ;{ }^{13} \mathrm{C}-\mathrm{NMR}\left(\mathrm{DMSO}-\mathrm{d}_{6}\right) \delta: 40.9\left(\mathrm{CH}_{2}\right), 68.3$ (CH), $103.6\left(\mathrm{CH}_{2}\right), 103.8\left(\mathrm{CH}_{2}\right), 104.9(\mathrm{CH}), 105.5(\mathrm{CH}), 107.2(\mathrm{CH}), 111.6(\mathrm{CH}), 129.6(\mathrm{C}), 138.0$ (C), 141.6 (C), 144.4 (C), 146.8 (C), 147.3 (C), 151.3 (C), 152.3 (C); Anal. Calcd for $\mathrm{C}_{16} \mathrm{H}_{12} \mathrm{~N}_{2} \mathrm{O}_{9}$ : C, 51.07; H, 3.21; N, 7.44. Found: C, 50.86; H, 3.13; N, 7.20.

1-(6-Nitrobenzo[1,3] dioxol-5-yl)-2-(4-nitrophenyl)propan-2-ol (4h): Orange solid; mp $99 \quad{ }^{\circ} \mathrm{C}$ (chloroform); ${ }^{1} \mathrm{H}-\mathrm{NMR}\left(\mathrm{CDCl}_{3}\right) \delta: 1.65$ (s, 3H, $\mathrm{CH}_{3}$ ), 2.74 (bs, $\left.1 \mathrm{H}, \mathrm{OH}\right), 3.24\left(\mathrm{~d}, J_{\mathrm{AB}}=13.9 \mathrm{~Hz}, 1 \mathrm{H}\right.$, $\mathrm{CH}_{2}$ ), $3.61\left(\mathrm{~d}, J_{\mathrm{AB}}=13.5 \mathrm{~Hz}, 1 \mathrm{H}, \mathrm{CH}_{2}\right), 6.06\left(\mathrm{~s}, 2 \mathrm{H}, \mathrm{CH}_{2}\right), 6.38\left(\mathrm{~s}, 1 \mathrm{H}, \mathrm{H}_{\mathrm{Ar}}\right), 7.39\left(\mathrm{~s}, 1 \mathrm{H}, \mathrm{H}_{\mathrm{Ar}}\right), 7.59(\mathrm{~d}$, $\left.J=8.9 \mathrm{~Hz}, 2 \mathrm{H}, 2 \mathrm{xH}_{\mathrm{Ar}}\right), 8.18\left(\mathrm{~d}, J=8.9 \mathrm{~Hz}, 2 \mathrm{H}, 2 \mathrm{xH}_{\mathrm{Ar}}\right) ;{ }^{13} \mathrm{C}-\mathrm{NMR}\left(\mathrm{CDCl}_{3}\right) \delta: 30.0\left(\mathrm{CH}_{3}\right), 45.6\left(\mathrm{CH}_{2}\right)$, $75.1(\mathrm{C}), 102.9\left(\mathrm{CH}_{2}\right), 105.8(\mathrm{CH}), 111.7(\mathrm{CH}), 123.5(2 \mathrm{xCH}), 126.1(2 x \mathrm{CH}), 127.8(\mathrm{C}), 144.7(\mathrm{C})$, 147.0 (C), 151.0 (C), 154.4 (C). The second C-nitro was not observed in this experiment; Anal. Calcd for $\mathrm{C}_{16} \mathrm{H}_{14} \mathrm{~N}_{2} \mathrm{O}_{7}$ : C, 55.49; H, 4.07; N, 8.09. Found: C, 55.51; H, 4.07; N, 7.89.

2-Hydroxy-2-methyl-3-(6-nitrobenzo[1,3] dioxol-5-yl)propionic acid ethyl ester (4i): Yellow solid; mp $79{ }^{\circ} \mathrm{C}$ (chloroform); ${ }^{1} \mathrm{H}-\mathrm{NMR}\left(\mathrm{CDCl}_{3}\right) \delta: 1.26$ (t, $\left.J=7.2 \mathrm{~Hz}, 3 \mathrm{H}, \mathrm{CH}_{3}\right), 1.41$ (s, 3H, $\mathrm{CH}_{3}$ ), 3.28 (bs, $1 \mathrm{H}$, $\mathrm{OH}), 3.35\left(\mathrm{~d}, J_{\mathrm{AB}}=13.9 \mathrm{~Hz}, 1 \mathrm{H}, \mathrm{CH}_{2}\right), 3.43\left(\mathrm{~d}, J_{\mathrm{AB}}=13.9 \mathrm{~Hz}, 1 \mathrm{H}, \mathrm{CH}_{2}\right), 4.12(\mathrm{q}, J=7.2 \mathrm{~Hz}, 2 \mathrm{H}$, $\mathrm{CH}_{2}$ ), 6.04 (s, 2H, $\left.\mathrm{CH}_{2}\right), 6.81$ (s, $\left.1 \mathrm{H}, \mathrm{H}_{\mathrm{Ar}}\right), 7.31$ (s, $\left.1 \mathrm{H}, \mathrm{H}_{\mathrm{Ar}}\right) ;{ }^{13} \mathrm{C}-\mathrm{NMR}\left(\mathrm{CDCl}_{3}\right) \delta: 13.9\left(\mathrm{CH}_{3}\right), 26.0$ $\left(\mathrm{CH}_{3}\right), 40.8\left(\mathrm{CH}_{2}\right), 62.3\left(\mathrm{CH}_{2}\right), 74.8(\mathrm{C}), 102.7\left(\mathrm{CH}_{2}\right), 105.6(\mathrm{CH}), 111.6(\mathrm{CH}), 126.9(\mathrm{C}), 144.7(\mathrm{C})$, 146.7 (C), 150.6 (C), 175.9 (C); Anal. Calcd for $\mathrm{C}_{13} \mathrm{H}_{15} \mathrm{NO}_{7}$ : C, 52.53; H, 5.09; N, 4.71. Found: C, 52.51; H, 5.08; N, 4.81.

2-Hydroxy-3-(6-nitrobenzo[1,3]dioxol-5-yl)-propionic acid ethyl ester (4j): Yellow solid; mp $90{ }^{\circ} \mathrm{C}$ (chloroform); ${ }^{1} \mathrm{H}-\mathrm{NMR}\left(\mathrm{CDCl}_{3}\right) \delta: 1.30\left(\mathrm{t}, J=7.2 \mathrm{~Hz}, 3 \mathrm{H}, \mathrm{CH}_{3}\right), 2.94$ (bs, $\left.1 \mathrm{H}, \mathrm{OH}\right), 3.08\left(\mathrm{dd}, J_{\mathrm{AB}}=\right.$ 
$13.8 \mathrm{~Hz}$ and $J=8.5 \mathrm{~Hz}, 1 \mathrm{H}, \mathrm{CH}_{2}$ ), 3.45 (dd, $J_{\mathrm{AB}}=13.8 \mathrm{~Hz}$ and $\left.J=4.3 \mathrm{~Hz}, 1 \mathrm{H}, \mathrm{CH}_{2}\right), 4.24(\mathrm{q}, J=7.2$ $\left.\mathrm{Hz}, 2 \mathrm{H}, \mathrm{CH}_{2}\right), 4.47$ (m, 1H, CH), 6.09 (s, 2H, $\left.\mathrm{CH}_{2}\right), 6.83$ (s, $\left.1 \mathrm{H}, \mathrm{H}_{\mathrm{Ar}}\right), 7.48\left(\mathrm{~s}, 1 \mathrm{H}, \mathrm{H}_{\mathrm{Ar}}\right) ;{ }^{13} \mathrm{C}-\mathrm{NMR}$ $\left(\mathrm{CDCl}_{3}\right)$ \&: $14.1\left(\mathrm{CH}_{3}\right), 37.9\left(\mathrm{CH}_{2}\right), 62.2\left(\mathrm{CH}_{2}\right), 70.4(\mathrm{CH}), 102.9\left(\mathrm{CH}_{2}\right), 105.7(\mathrm{CH}), 111.9(\mathrm{CH})$, 128.9 (C), 143.6 (C), 147.0 (C), 151.4 (C), 174.1 (C); Anal. Calcd for $\mathrm{C}_{12} \mathrm{H}_{13} \mathrm{NO}_{7}$ : C, 50.89; H, 4.63; N, 4.95. Found: C, 50.90; H, 4.62; N, 4.89.

2-Hydroxy-2-(6-nitrobenzo[1,3]dioxol-5-yl-methyl) malonic acid diethyl ester (4k): Orange solid; mp $92{ }^{\circ} \mathrm{C}$ (chloroform); ${ }^{1} \mathrm{H}-\mathrm{NMR}\left(\mathrm{CDCl}_{3}\right) \delta: 1.26$ (t, $\left.J=7.2 \mathrm{~Hz}, 6 \mathrm{H}, 2 \mathrm{xCH}_{3}\right), 3.76\left(\mathrm{~s}, 2 \mathrm{H}, \mathrm{CH}_{2}\right), 3.80$ (bs, $1 \mathrm{H}, \mathrm{OH}), 4.22$ (q, $\left.J=7.2 \mathrm{~Hz}, 4 \mathrm{H}, 2 \mathrm{xCH}_{2}\right), 6.06\left(\mathrm{~s}, 2 \mathrm{H}, \mathrm{CH}_{2}\right), 6.90\left(\mathrm{~s}, 1 \mathrm{H}, \mathrm{H}_{\mathrm{Ar}}\right), 7.32\left(\mathrm{~s}, 1 \mathrm{H}, \mathrm{H}_{\mathrm{Ar}}\right) ;{ }^{13} \mathrm{C}-$ NMR $\left(\mathrm{CDCl}_{3}\right)$ \&: $13.9\left(2 \mathrm{xCH}_{3}\right), 35.6\left(\mathrm{CH}_{2}\right), 62.9\left(2 \mathrm{xCH}_{2}\right), 78.7(\mathrm{C}), 102.7\left(\mathrm{CH}_{2}\right), 105.7(\mathrm{CH}), 111.8$ (CH), $125.4(\mathrm{C}), 147.0(\mathrm{C}), 150.7$ (C), 169.5 (2xC). The C-nitro was not observed in this experiment; Anal. Calcd for $\mathrm{C}_{15} \mathrm{H}_{17} \mathrm{NO}_{9}$ : C, 50.71; H, 4.82; N, 3.94. Found: C, 51.07; H, 4.88; N, 3.99.

3-Hydroxy-1-methyl-3-(6-nitrobenzo[1,3] dioxol-5-ylmethyl)-1,3-dihydroindol-2-one (4l): Yellow solid; mp $161{ }^{\circ} \mathrm{C}$ (chloroform); ${ }^{1} \mathrm{H}-\mathrm{NMR}\left(\mathrm{CDCl}_{3}\right) \delta$ : 2.79 (bs, $\left.1 \mathrm{H}, \mathrm{OH}\right), 3.16\left(\mathrm{~s}, 3 \mathrm{H}, \mathrm{CH}_{3}\right), 3.35\left(\mathrm{~d}, J_{\mathrm{AB}}=\right.$ $\left.13.9 \mathrm{~Hz}, 1 \mathrm{H}, \mathrm{CH}_{2}\right), 3.74\left(\mathrm{~d}, J_{\mathrm{AB}}=13.9 \mathrm{~Hz}, 1 \mathrm{H}, \mathrm{CH}_{2}\right), 6.10\left(\mathrm{~s}, 2 \mathrm{H}, \mathrm{CH}_{2}\right), 6.82\left(\mathrm{~m}, 1 \mathrm{H}, \mathrm{H}_{\mathrm{Ar}}\right), 6.91(\mathrm{~s}, 1 \mathrm{H}$, $\mathrm{H}_{\mathrm{Ar}}$ ), $7.03\left(\mathrm{~m}, 2 \mathrm{H}, 2 \mathrm{xH}_{\mathrm{Ar}}\right), 7.31\left(\mathrm{~m}, 1 \mathrm{H}, \mathrm{H}_{\mathrm{Ar}}\right), 7.45$ (s, $\left.1 \mathrm{H}, \mathrm{H}_{\mathrm{Ar}}\right) ;{ }^{13} \mathrm{C}-\mathrm{NMR}\left(\mathrm{CDCl}_{3}\right) \delta: 26.3\left(\mathrm{CH}_{3}\right), 40.7$ $\left(\mathrm{CH}_{2}\right), 76.1(\mathrm{C}), 102.9\left(\mathrm{CH}_{2}\right), 105.9(\mathrm{CH}), 108.5(\mathrm{CH}), 112.5(\mathrm{CH}), 123.1(\mathrm{CH}), 123.9(\mathrm{CH}), 126.5(\mathrm{C})$, 129.4 (C), 129.9 (C), 142.9 (C), 144.1 (C), 147.2 (C), 151.1 (C), 177.2 (C); Anal. Calcd for $\mathrm{C}_{17} \mathrm{H}_{14} \mathrm{~N}_{2} \mathrm{O}_{6}$ : C, 59.65; H, 4.12; N, 8.18. Found: C, 59.57; H, 4.19; N, 8.03.

\section{References}

1 Ruffolo, R.R.; Bondinell, W.; Hieble, J.P. J. Med. Chem. 1995, 38, 3681-3716.

2 Briscoe, M.W.; Chambers, R.D.; Mullins, S.J.; Nakamura, T.; Vaughan, J.F.S. ; Drakesmith, F.G. J. Chem. Soc. Perkin. Trans. 1, 1994, 3115-3118.

3 Burkholder, C.; Dolbier, Jr W.R.; Médebielle, M. J. Org. Chem. 1998, 63, 5385-5394.

4 Médebielle, M.; Kato, K.; Dolbier, Jr W.R. Synlett. 2002, 1541-1543.

5 Giuglio-Tonolo, G.; Terme, T.; Médebielle, M.; Vanelle, P. Tetrahedron Lett. 2003, 44, 64336435.

6 Giuglio-Tonolo, G.; Terme, T.; Médebielle, M.; Vanelle, P. Tetrahedron Lett. 2004, 45, 51215124.

$7 \quad$ Vanelle, P.; Terme, T.; Gellis, A.; Crozet, M. P. ; Res. Adv. Org. Chem. 2000, 1, 27-41.

8 Vanelle, P.; Terme, T.; Crozet, M.P. Recent Res. Devel. Organic Chem. 2001, 5, 129- 150.

9 Terme, T.; Crozet, M.P.; Maldonado, J.; Vanelle, P. In Electron Transfer Reactions in Organic Synthesis, P. Vanelle, Ed.; Research Signpost: Trivandrum, India, 2002; pp. 1-42.

10 Meuche-Albeny, J.; Rathelot, P.; Crozet, M.P.; Vanelle P. Chem. Heterocycl. Compounds 2003, 8, 1139-1147.

Sample Availability: Available from the authors.

(C) 2005 by MDPI (http://www.mdpi.org). Reproduction is permitted for noncommercial purposes. 